\title{
THE ANNUAL MEETING IN SAN ANTONIO
}

The seventy-sixth annual meeting of the American Mathematical Society was held at the San Antonio Convention Center in San Antonio, Texas on Thursday, January 22 through Sunday, January 25, in conjunction with the Mathematical Association of America. There were 3994 registrants, including 2603 members of the Society.

The forty-third Josiah Willard Gibbs Lecture, Tides and time, was delivered by Professor Walter H. Munk of the University of California, San Diego at 8:00 p.m. on Thursday, January 22. Professor Munk was introduced by Professor H. S. MacDonald Coxeter, Vice President of the Society.

By invitation of the Committee to Select Hour Speakers for the Summer and Annual Meetings, there were two invited addresses. Professor Morris W. Hirsch of the University of California, Berkeley, gave an address entitled Foliations and noncompact transformation groups. He was introduced by Professor John Milnor of the Massachusetts Institute of Technology. Professor Daniel R. McMillan of the Institute for Advanced Study and the University of Wisconsin spoke on Acyclicity in 3-manifolds. He was introduced by Professor R. H. Bing of the University of Wisconsin.

Two Cole Prizes were awarded on Friday, January 23rd, one to Professor John Stallings of the University of California at Berkeley and the other to Professor R. G. Swan of the University of Chicago. The prizes were given in recognition of their work as follows: John Stallings, On torsion free groups with infinitely many ends, Annals of Math., Vol. 88, No. 2, Sept., 1968, pp. 312-334; R. G. Swan, Groups of cohomological dimension 1, Journal of Algebra, Vol. 12 (1969), pp. 585-610.

There were sixty-four sessions for contributed ten-minute papers, presided over by the following: Paul B. Bailey, H. L. Bentley, Kenneth R. Berg, Harry W. Berkowitz, Russell G. Bilyeu, Henry W. Block, J. C. Bradford, Dennison R. Brown, Ezra Brown, James Raymond Bunch, Philip Calabrese, J. H. Carruth, Donald R. Chalice, Joseph A. Cima, Charles V. Coffman, Craig Comstock, Roger Cooper, Frederick H. Croom, Wayne Cutrer, David F. Dawson, Frank R. Demeyer, James Dorroh, Walter Gautschi, Robert Gordon, Jack E. Graver, Lino Gutierrez-Novoa, Charles Holmes, Peter G. Jessup, James S. Johnson, Steven L. Jordan, Vadim Komkov, S. Kinoshita, Richard B. Lavine, Wolfgang J. Liebert, M. H. Martin, Richard K. Miller, Theodore Motzkin, Sheldon E. Newhouse, William R. Nico, Charles McArthur, Lavon B. Page, Edgar M. Palmer, Donald B. 
Parker, Stanley E. Payne, C. R. Pittman, Marion C. Rayburn, John M. Saade, Donald G. Saari, Steven Schonefeld, Joel E. Schneider, Walter J. Schneider, Shawky E. Shamma, Robert A. Shive, Warren E. Shreve, Oberta A. Slotterbeck, Frank A. Smith, William H. Sumners, J. Dalton Tarwater, Leopoldo V. Toraballa, Jerry E. Vaughn, Ronald L. Wagoner, Charles N. Winton, Miccael J. Wright, E. C. Young.

Abstracts of papers presented at the meeting appear in the Notices of the American Mathematical Society for January, 1970.

The Arrangements Committee for the meeting consisted of D. S. Nymann, chairman, H. L. Alder, ex officio, W. E. Christilles, O. G. Harrold, Jr., ex officio, W. E. Hunt, Peter Terwey, Jr. (vice chairman), G. L. Walker, ex officio.

The Council met on January 21, 1970 at 2:00 P.M. in the Cavalier Room of the Hilton Hotel in San Antonio, with Vice President Coxeter in the chair.

The Council was informed that the Executive Committee had set a meeting for October 31, 1970 in Washington, D. C. and that the meeting already authorized for the Chicago area in the spring of 1971, preferably at a university, would be held on March 26-27, 1971 at the Chicago Circle Campus of the University of Illinois.

The Council accepted an invitation from the California Institute of Technology for the Society to meet in Pasadena on November 21, 1970.

The following persons were representatives of the Society at various functions as noted:

Don Mittleman at the 125th Anniversary Celebration at St. Mary's College

Charles D. Robinson at the inauguration of John C. Stevens as President of Abilene Christian College

Francis C. Hatfield at the inauguration of Frank R. Barth as President of Gustavus Adolphus College

William S. Mahavier at the inauguration of Arthur G. Hansen as President of Georgia Institute of Technology

Morris Marden at the inauguration of Charles Vevier as President of Adelphi University

Elsie Muller at the inauguration of Richard L. Bowen as President of the University of South Dakota

Burton W. Jones at the Centennial Founders' Day Convocation and inauguration of A. R. Chamberlain as President of Colorado State University. 
President Zariski has established committees and has made appointments to committees as noted:

Committee to Select Hour Speakers for Southeastern Sectional Meetings: Gail Young

Committee to Select Hour Speakers for Eastern Sectional Meetings: G. A. Hunt

Committee to Select Hour Speakers for Far Western Sectional Meetings: Ronald Getoor

Committee to Select Hour Speakers for Summer and Annual Meetings: J. K. Moser

Committee to Monitor Problems in Communication: F. P. Peterson, Allen Shields

Committee on Opportunities in Mathematics for Disadvantaged Groups: Raymond Ayoub, Chairman, Llayron L. Clarkson, Gloria Gilmer, Richard K. Lashof, Cathleen Morawetz, David A. Sánchez, C. T. Taam

Committee on Financial and Legal Problems of Supporting Withdrawal of Service: Wendell Fleming, Murray Gerstenhaber, J. T. Schwartz, Chairman

Committee to Advise on Analysis of Employment Data: R. D. Anderson, William L. Duren, Jr., Chairman, Gail Young

Committee to Select the Gibbs Lecturers for 1971 and 1972: Mark Kac, Chairman, J. J. Stoker, R. L. Wilder

Committee to Select the Winner of the Veblen Prize: R. Bott, Chairman, Morton Brown, S. Smale

Committee on Summer Institutes: J. L. Doob

Tellers for 1969 Election: Jerry L. Kazdan, Norman Oler

Arrangements Committee for the Annual Meeting of 1970 in San Antonio: D. S. Nymann, Chairman, H. L. Alder, ex officio, W. E. Christilles, O. G. Harrold, Jr., ex officio, W. E. Hunt, Peter Terwey, Jr., G. L. Walker, ex officio

Arrangements Committee for the Summer Meeting of 1970 in Laramie: H. L. Alder, ex officio, Roger M. Cooper, Rev. Fred T. Daly, George C. Gastl, Joanne George, John H. George, William C. Guenther, J. Ray Hanna, Terry L. Jenkins, Joe E. Kirk, Jr., Robert W. McKelvey, R. S. Pierce, ex officio, A. Duane Porter, John H. Rowland, W. Norman Smith, Chairman, P. O. Steen, James W. Thomas, G. L. Walker, ex officio, Laurence Weinberg

Advisory Committee for the Sixth Berkeley Symposium on Mathematical Statistics: Steven Orey 
AMS-IMS Committee on Translations from Russian and Other Foreign Languages: A. H. Wallace

Liaison Representative with ACM 70 Year: A. H. Taub.

The Council received the reports of Executive Committee Business by Mail stating that the following 321 nominees of institutional members were elected to membership:

Arizona State University: Jerry R. Ridenhour.

University of Arkansas: Dayne Turner Hogan.

Auburn University: Jane I. Brown, James Campbell Morgan, George Michael Reed. University of British Columbia: Gin Hor Chan, Frederic Arthur Ferdman, Ming Huat Lim, Ping K. Tam, Korkikeong Tan, Edward T. H. Wang, John C. Wells, Kenneth Wayne Welsh.

Brooklyn College: Charles S. Kleinberg.

Brown University: R. Daniel Bergeron, J. E. Kriegel, Alan Levine, William R. Melvin, Abdulimajid Nosayr.

Bucknell University: H. Lamar Bentley, Kenneth S. Kaminsky, Christopher R. Schell. California State College at Dominguez Hills: Chi-Lung Chang, Gordon Matthews.

California State Polytechnic College: Lynn M. Cummins, Bruce Russell Gove, Ronald L. Jepsen.

University of California at Berkeley: John Thomas Gill, George F. McNulty, Alan Saleski, Mehrdad M. Shahshahani, Laurence R. Taylor, Juan Alfredo Tirao, Douglas J. Young.

University of California at Davis: Wei Yung Chang, Dean H. Fearn, Bob Wai-Man Lau, Joan Machiz, Barry Schiller.

University of California at Riverside: Willie B. Avant, Jr.

University of California at San Diego: Leo B. Cashman, Tunc Geveci.

University of California at Santa Barbara: Chung-Wei Ha, Spyridon E. Therianos, Charles L. Thorne.

University of California at Santa Cruz: Estelle L. Cranford, Robert Lawrence Dubinsky, Daniel Friedman.

University of Chicago: John James Currano.

Clark University: Elizabeth Ramsay Clark, Clifford L. Pellftier.

Clarion State College: Irvin C. Kenny, Ronald A. Kapas, Thomas V. Wimer.

Columbia University: Phillis A. Arnold, Eric John Braude, Morris Goldfeld, John Johnson, George R. Kempf, D. Laksov, Jeanine M. Meyer, Makoto Namba, Chung-Kong A. Poon, Clyde B. Schechter, William S. Sit, Man Keung Siu, Duraiswamy Sundararaman, Torgny Svanes, Joseph Tomasovic.

Cornell University: Sampson O. Akuoko, Richard C. Eells, Larry R. Etzweiler, Laura

F. Farkas, Ilan Kozma, Chao-Chu Liang, George Metakides, Richard S. Millman, Leon P. Polek, Bjorn Schellenberg, Alan D. Sloan, Fred Solomon, James W. Thomas, Domingo Toledo, Samuel S. Wagstaff, Jr.

Dallas Baptist College: Mildred M. Mitchell, Marsha K. Pool, Charles D. Robbins.

DePaul University: Daniel E. Fyock, George Howard Litman, Joseph Ly.

Drexel Institute of Technology: Otha L. Britton, John H. Staib.

Duke University: Thomas R. Kramer.

Florida Presbyterian College: Paul C. Gruenberg, Jr.

Harvard University: Andreas Raphael Blass, Jerry Lloyd Bona, Peter B. Gilkey, Robert B. MacPherson. 
University of Hawaii: Judith AuHoy, Kazuo Ishizaka, Johannes A. Van Casteren.

Hope College: Herbert L. Dershem, Dean D. Sommers.

University of Houston: Betty Jane Barr, Larry L. Hinman.

Idaho State University: Robert H. McElfresh, Sharon F. Mohning, William F. Sanger.

Illinois Institute of Technology: Phyllis M. Kittel.

Indiana University: Hardip Singh Ahluwalia, Joel H. Anderson, Neela Bhatia, John

M. Cauffman, Robert R. Crawford, C. Michael Fox, Prazibha Gajendragadkar, John P. Hesemann, J. C. Kelly, John S. Lancaster, J. M. Osterburg, J. Brendan Quigley, Mary Rayar, Mohan Sharad Shrikhande, Bhushan Lal Wadhwa, Tinikin Wong, H. Zand.

Ithaca College: Shirley O. Hockett.

Johns Hopkins University: Chungming An, Jeffrey Ralph Butz, David L. Deal, John H. Dineen, Lawrence C. Gray, Robert W. Hesselbacher, Jr., Wilburn Clay Hoskins, Tsong-jen Huang, Alan W. Hunsberger, Chung-Yuan Cyma Lin.

Kent State University: Hsien-chung Lin.

Lakehead University: Douglas J. R. McKessock, Dietrich W. Schwagerl, Geraldine S. Service.

Lehigh University: Damir Franekic, Gerald M. Higdon, Brian J. Kelly, John F. Miller, Steve M. Rohde, Donald A. Thompson.

University of Louisville: Jo Ann Schmalz, Barbara F. Whitfield.

Loyola College, Baltimore: Gregory Nicholas Fiore, Robert M. Gaare.

Marquette University: Aloysius A. Sieracki, Leonard F. Klosinski.

Massachusetts Institute of Technology: Jon H. Davis, Hermann Flaschka, Robert Mandl, David L. Myers, Tomas P. Schonbek.

McMaster University: Vasagamoorthi Krishnasamy.

Memorial University of Newfoundland: Glenn W. Collins, Lakshmi Shanker Dube, Eric John Henry Moore.

University of Miami: David M. Morris.

Michigan State University: William G. Chang, Charles R. Diminnie, Michael Jon Evans, Richard A. Gibbs, Francis L. Jones, Steven J. Leon, Stephen C. Littell, John Arthur Oman, Louis T. Richards, Kathleen A. Taylor, S. Christelle, J. Edward Tornga.

University of Michigan: Rashid Ahman, Alan Kaylor Cline, Kenneth E. Guire, Joseph Hesse, Dost Mohammed Khan, Alan L. MacDonald, Victor T. Norton, Jr., Bruce P. Palka, Norberto Salinas.

Millersville State College: James Bobeck, Diane Miller, Robert Schluben.

University of Missouri: James R. Clark

Monmouth College: Marvin H. Goodman, Richard H. Guilfoyle.

Morehouse College: Robert E. Brown, Curtis Clark, Claude Robinson, Jr.

Naval Postgraduate School: Ronald A. Rinaldi, Orjal L. Sweeney.

Newark College of Engineering: Kenneth L. Kaufman.

Nerw Mexico State University: Edward E. Ferguson, Dave E. Peercy, David G. Tabor.

University of New Mexico: Sarah L. M. Christiansen, Ih Ching Hsu.

North Carolina State University: James C. Halsey, Lawrence M. Hanafy, Daniel W. Krider, Jo Perry Ledbetter, Gerald A. Marin, Chao-Kuang Pian, Richard M. Willett.

University of North Carolina-Chapel Hill: John W. Losse.

University of North Carolina-Charlotte: Stephen E. Fuller, John Leslie Henderson, Jane Stein.

University of North Carolina-Greensboro: Dargan Frierson, Jr. 
Northwestern University: Klavs G. Fischer.

Oberlin College: John Charles Henze, William George Fleissner, John L. Miller.

Ohio Wesleyan University: Joan Ericson Smoot.

Olivet College: Elaine Wellman Becker.

Oregon State University: Keane B. McGee, Jr.

University of Ottawa: Sanjiv Rangachari, Cheuk F. Wong.

Pace College: Stephen L. Berman, Zeljko Gregov.

Pennsylvania State University: Frank Ralph Dangello, Garry Brian Green, Webb C. Miller.

University of Pennsylvania: Michael O. Albertson, David M. Berman, Maurice J. Duprf, William Lohr Green, Alan Hopenwasser, Preston Kohn, Richard J. Kramer, Kenneth Krigelman, Michael R. Levy, Peter Norman, T. J. O'Malley, Jane C. Purcell, Hadas Rin, Robert A. Rubin, Steven I. Sperber, Jorgen Vesterstrom, Robert Clark Whitton, Sandra Zaroodny.

Polytechnic Institute of Brooklyn: Gerard Christopher Murphy, Richard Rosenthal. University of Pittsburgh: John J. Buoni, James W. Kennedy

University of Puerto Rico: Rafael R. Arias, Martha Luisa Tapia, Gilda Elena VicentiCernuda.

University of Rhode Island: Richard H. Lavoie.

University of Rochester: Joseph Peter Duran, Douglas A. Gale, Ming-Jung Lee.

Saint Francis College: James W. Lieb, Richard J. Parrish.

San Fernando Valley State College: Robert D. Grone, Karl B. Hagemann.

San Francisco State College: Richard A. Levaro, M. Shimada, Ray Karl Westergard. University of Santa Clara: David A. Hesson, Robert W. Owens, Daniel C. White.

University of Saskatchewan: Murli Manohar Gupta.

University of the South: Dwight E. Davis, Stephen F. Lelaurin, Michael S. Pindzola.

Southern Illinois University-Edwardsville: Bess A. Eastin, James L. Hindflang, Kenneth E. Stenzel.

Southwest Texas State University: Charles J. Campbell, Linda Ruth Freeman.

State University of New York at Albany: David J. Hallenbeck, Sally A. Malik, Ann M. Penton.

State University of New York-College at Oswego: K. Michael DeLorm.

Susquehanna University: John M. Reade III, Margaret Anne Rogers, Wallace J. Growney.

Texas $A \mathcal{E}^{\circ} M$ University: James A. Graves.

University of Toledo: Martha Anker, Gene William Arnold.

Trinity College: Victor R. Levine, Robert C. Moore.

Tufts University: Satish A. Kalavase, Sister Barbara J. Loud, Eric Myrvaagnes.

Tulane University: Roger D. Bleier, Donald A. Chambless, Hugh M. Collins, Danny J. Moore.

University of Tulsa: Yun Hwang Boo, Joy Yvonne Starks.

Union College: Douglas Hatch, Dean G. Hoffman.

Upsala College: C. Lincoln Brown, Robert M. Reed, Ross Nelson Schneider.

Vanderbilt University: Baxter Johns, Ronald C. Linton, Chilin Yen.

Virginia Commonwealth University: William Lee Hoard.

Wake Forest University: Daniel J. Richman.

Washington State University: Richard G. Burton, Eugene Iwao Furuyana, Reginald D. Laursen.

University of Washington: Lawrence J. Brenton, James C. Davies, Andrew J. H. Vogt. Wayne Slate University: Linda Wells Brinn. 
Wesleyan University: Wayne M. Lawton.

Western Washington State College: Ronald W. Engstrom, Leo C. Maki. Williams College: Henry M.Walker.

University of Wisconsin-Madison: Theodore J. Hartman.

University of Wyoming: Dwight M. Olson, Yako L. Varol, Arthur F. Wright.

The following persons have been admitted to the Society in accordance with reciprocity agreements with various mathematical organizations:

Australian Mathematical Society: Peter William Aitchison, B. J. Day, Jack D. Gray, Walter D. Neumann, Alfred J. Van Der Poorten, Walter D. Wallis, Robert F. C. Walters.

Deutsche Mathematiker Vereinigung: Hubert Berens, Horst Herrlich, K. W. Kirchgassner, Radu Theodorescu.

Edinburgh Mathematical Society: James Ford, Sheila A. McKilligan.

Indian Mathematical Society: N. Ganeson, Subhas Chandra Gupta, J. G. Krishna, I. B. S. Passi, Romesh Singh.

Israel Mathematical Union: Dov Aharonov.

London Mathematical Society: Ronald Brown, John William S. Cassells, Roy O. Davies, H. K. Farahat.

Mathematical Society of Japan: Takateru Okayasu, Akihro Okuyama, T. Otsuki, Kazuyuki Saito, Onodera Takeski, Akira Tsutsumi.

Sociedade de Matematica de Sao Paulo: C. M. De Barros.

Sociedad Matematica Mexicana: Jo L. Arraut.

Societe Mathematique De Belgique: Ludo C. M. C. Buyst, Jean Doyen, Jacques V. Maes.

Societe Mathematique de France: A. Gauderoy, Gerard Tronel.

Svenska Matematikersamfundet: Carl-Otto Segerdahl.

Union Matematica Argentina: J. M. Alonso.

Wiskundig Genootschap Te Amsterdam: Nelly Verbeek-Kroonenberg.

The Secretary announced the election of the following 159 persons to ordinary membership:

Abrams, Robert A., University of Illinois;

Agarwal, Arun Kumar, Grambling College;

Allen, G. D.

Anderson, Larry R., Whitman College;

Barkhurst, Jon R., 842 Hardingway E., Galion, Ohio;

Behara, M., McMaster University;

Berman, Elizabeth A., 3016 W 73rd Street, Prairie Village, Kansas;

Blake, Louis H., Northern Illinois University;

Bliedtner, Jurgen E., Yale University;

Bodenrader, Joseph C., State University of New York, College at Plattsburgh;

Bolis, Theodore S., 1111 North Dartmouth, Claremont, California;

Bonneau, Richard J., Raytheon, Sudbury, Massachusetts;

Bradley, Gordon H., Yale University;

Briggs, William J., University of Pennsylvania;

Brodsky, Stuart L., U. S. Naval Ordnance Laboratories, Silver Spring, Maryland;

Bronson, Richard D., Fairleigh Dickinson University;

Brown, Ezra, Virginia Polytechnic Institute; 
Brown, Wayland Yoder, Jr., Armstrong State College;

Brunner, H., Dalhousie University;

Bukovsky, Lev, Saearik University, Kosice, Czechoslovakia;

Burns, John W., U. S. Army Medical Research Laboratories, Fort Knox, Kentucky;

Butts, Hubert S., Louisiana State University;

Carter, Lyne Hyer, Florida Institute of Technology;

Chang, Long F., Owen Illinois, Toledo, Ohio;

Chelak, Alex R., Hoffmann-LaRoche, Nutley, New Jersey;

Chi, Lan Keh, RCA, Riverton, New Jersey;

Cohen, Martin A., Singer, General Precision, Wayne, New Jersey;

Cohen, Robert, York College;

Coleman, Norman P., Jr., Rock Island Arsenal, Rock Island, Illinois;

Connett, John E., Northern Illinois University;

Conroy, John E., Eglin Air Force Base, Fort Walton Beach, Florida;

Crestin, Jean-Pierre, ENSTA, Paris, France;

Davidson, James Ross, U. S. Air Force Academy;

Davis, Clyde W., Jr., Columbus College;

Deitch, Robert David, 23 E. Ohio Street, No. 308, Indianapolis, Indiana;

DeLibero, F. E., 30 Kewis Road, North Merrick, New York;

Dowling, Thomas A., University of North Carolina-Chapel Hill;

Eggen, Dale Royce, 2828 Palm Springs Way, Las Vegas, Nevada;

Evans, Kenneth A., Southwest Texas State University;

Finkelstein, Raphael P., Bowling Green State University;

Fischer, Addison M., West Virginia University;

Forgham, James Louis, 3595 Lighthouse Drive, Palm Beach Gardens, Florida;

Forgue, Vernon R., 2322 Russell Street, Berkeley, California;

Frank, James L., Virginia Polytechnic Institute;

Fraser, Grant A., University of California at Los Angeles;

Fulton, David Leroy, University of Connecticut;

Ganguly, Anil K., Advanced Teacher's College, Zaria, Nigeria;

Geiger, David S., University of Illinois;

Ghoshal, Sudhanshu Kumar, University of Toronto;

Goldberg, Merrill B., University of Colorado;

Gorton, Robert Bruce, University of Dayton;

Greene, Peter H., 1640 East 50th Street, Chicago, Illinois;

Grieser, David R., Rocky Hill School, East Greenwich, Rhode Island;

Groh, Hans Joachim C., Lakehead University;

Guillou, Louis A., St. Mary's College;

Hadwin, Donald W., Augustana College;

Hale, Douglas F., Ohio State University;

Hangan, Theodor, Institute of Math, Bucuresti, Romania;

Haynes, John William, Litton Industries, Woodland Hills, California;

Hecht, Ben, University of South Carolina;

Heiberg, Charles H., Ohio State University;

Hern, Thomas A., Ohio State University;

Heuvers, Konrad John, Michigan Technological University;

Hicks, Darrell Lee, Sandia Laboratories, Albuquerque, New Mexico;

Horr, Donald E., Lincoln College;

Hyduke, D. J., Susquehanna Corporation, Alexandria, Virginia;

Ibele, Charles E., U. S. Army;

Ivanoff, Vladimir F., 1149 Elm Street, San Carlos, California; 
Jaffe, Arthur Michael, Harvard University;

Jarvis, Roger G., Atomic Energy of Canada, Charles River, Ontario, Canada; Juelg, Patricia Ann, Corpus Christi Independent School District;

Kaczynski, Theodore J., 463 N. Ridge, Lombard, Illinois•

Kay, Alvin J., San Jacinto College;

Kellett, John M., Gettysburg College;

Kelly, Jerry Stewart, Syracuse University;

Kem, Margaret A., Golden Gate College;

Kemp, L. Franklin, Pan American Petroleum, Tulsa, Oklahoma;

Kent, George Alan, Brown University;

Kerrick, Jerrill D., Oregon State University;

Lemay, William H., Pennsylvania University, McKeesport Campus;

Liang, Joseph Jen-Yin, California Institute of Technology;

Littau, Marianne M., Mundelein College;

Maarschalk, Dennis, Fairleigh Dickinson University;

Malkus, Willem V. R., Massachusetts Institute of Technology;

Marek, Ivo K., Case Western Reserve University;

Martin, Robert H., Jr., Georgia Institute of Technology;

Mazeres, Reginald M., Tennessee Technological University;

McLaurin, Johnnie W., University of California at Los Angeles;

McNeil, Vincent G., Bunker Ramo, Canoga Park, California;

Mertes, Sandra A., Catholic University of America;

Miller, Forrest R., Jr., Kansas State University;

Murphy, Patricia Ann, Maryland State College;

Myers, Dale W., University of California;

Nespoli, Edmund A., Harcourt Brace and World, New York;

Niman, John, Hunter College;

Ninomiya, Nobuki, Osaka City University, Japan;

Nuber, John Arthur, University of Miami;

O'Connor, Joel Leslie, University of Florida;

Oden, J. Tinsley, University of Alabama;

O'Mahony, Rosalie Mary, College of San Mateo;

Osterweil, Leon J., University of Maryland;

Padgett, Robert P., Xavier University;

Park, Jong Guen, Yonsei University, Seoul, Korea;

Parker, Elwood G., Guilford College;

Patrick, Alfred J., Ohio University;

Payton, Robert G., Adelphi University;

Perez-Olguin, Juan Luis, University of Chile;

Perry, Margaret R., American University;

Phelps, Dean, 1800 N.W. 4th Street, Apt. 58F, Gainesville, Florida;

Pla, Jean-Marie, 105 Bd suchet, Paris, France;

Poss, Samuel, City College of New York;

Potter, Meredith W., Kalamazoo College;

Potts, James A., Transamerica Insurance, Memphis, Tennessee;

Powers, Morris Walton, University of Alabama in Huntsville;

Ranade, Mary Stephens, Illinois Institute of Technology;

Rarback, Ann Stehney, State University of New York at Stony Brook;

Reilly, Robert L., Jr., Batten, Barton, Durstine, and Osborn, New York;

Robbins, Fred E., U. S. Underwater Sound Laboratories, New London, Connecticut; 
Rockwell, Richard D., Pacific Union College;

Rodriguez, Olga, University of Puerto Rico;

Roman, Steven M., University of California at Los Angeles;

Royse, James R., San Francisco State College;

Rubenfeld, Lester A., Rensselaer Polytechnic Institute;

Rudd, Carole H., Shorter College;

Russo, Ray L., Louisiana State University in New Orleans;

Sackett, Gary G., University of New Mexico;

Sacrredote, George S., University of Illinois;

Schleusner, John W., West Virginia University;

Schmidt, Bertram A., Central Michigan University;

Schwartz, Edward Norman, Belfer Graduate School of Science;

Sidman, Bernard, Sylvania Electric;

Silber, Jack, Roosevelt University;

Sim, Soon Kiong, University of Western Ontario;

Simenauer, Ronald F., 33-05 92nd Street, Jackson Heights, New York;

Singh, Kirtan, St. Mary's University;

Smith, Harry F., University of Iowa;

Smith, Robert A., 100 Sharon Court, Laurel, Mayland;

Sobota, James Michael, Michigan State University;

Sonnenschein, Hugo Freund, University of Minnesota;

Stallard, Frank W., Georgia Institute of Technology;

Steinberg, Howard, University of Wisconsin;

Stevenson, Nell E., State University of New York at Binghampton;

Stinebrickner, Ralph P., State University of New York College at Potsdam;

Strom, Arne, University of Oslo;

Stumpff, H. Deith, Central Missouri State College;

Sullivan, Theodore F., University of Notre Dame;

Sussmann, Hector Jose, 251 Mercer Street, New York, New York

Swanson, Ann M., National Bureau of Standards;

Swan, Douglas A., Principia College;

Tabachnick, Murray, University of Calgary;

Tafel, Richard E., Hughes Aircraft, Los Angeles, California;

Takamatsu, Tsurukichi, Daito-Bunka University, Tokyo, Japan;

Thompson, James M., Lockheed, Burbank, California;

Tolar, Robert A., Colorado State University;

Toohey, Terrence J., 111 5th Avenue, New York, New York;

Tse, Enoch Chi-Wah, Eastern Michigan University;

Ulmer, Milton, Wesleyan University;

Vanderjagt, Donald W., Grand Valley State College;

Venkatararian, Rangachari, University of Manitoba;

Ventura, John R., Jr., 68 Luke Street, New Bedford, Massachusetts;

Voigt, Robert G., University of Maryland;

Wajda, Louis W., College of Southern Idaho;

Wenger, Robert B., University of Wisconsin-Green Bay;

Wieting, Thomas W., Reed College;

Wilbur, Ronald I., Hughes Aircraft, Fullerton, California;

Wolfe, Robert J., Long Island University;

$\mathrm{Yu}$, Dahsoong, University of Oklahoma;

Zummer, J. A., University of Waterloo, Waterloo, Ontario, Canada. 
The following institutions were elected to institutional membership:

Augustana College, Sioux Falls, South Dakota

Boise State College, Boise, Idaho

Dallas Baptist College, Dallas, Texas

Millsaps College, Jackson, Mississippi

Norwich University, Northfield, Vermont

Olivet College, Olivet, Michigan

United States Coast Guard Academy, New London, Connecticut

University of Lethbridge, Lethbridge, Alberta, Canada

University of Maryland, Baltimore, Maryland

University of Puerto Rico, Mayaguez, Puerto Rico

Valdosta State College, Valdosta, Georgia

Wisconsin State University, Whitewater, Wisconsin

The Council elected Raoul Bott and Calvin C. Moore to membership on the Executive Committee.

The Council recommended that the Society adhere to the International Council of Scientific Unions Abstracting Board.

The report of the Steele Prize Committee was accepted, including the wording of the public announcement on p. 583.

The Council received the information that the Trustees, at the request of the Council, have agreed to eliminate the charge for the initial block of reprints, recently instituted in instances where page charges are not paid.

The Council approved a proposal by the Committee on Mathematics in the Life Sciences for a Symposium on Some Mathematical Questions in Biology to be held with joint sponsorship of the Society for Industrial and Applied Mathematics in conjunction with the December 1970 meeting of the American Association for the Advancement of Science in Chicago.

The Executive Director introduced the new Deputy Executive Director, Dr. Lincoln K. Durst, now Professor at the Claremont Men's College. He assumes his duties in the Summer of 1970.

In response to an invitation from President Longenecker of Tulane University, the Council "authorized Vice President Coxeter to appoint a committee to visit with the administration in an effort to resolve the conflict arising from the Dubinsky case." The Committee, subsequently appointed, consists of A. A. Albert, R. H. Bing, and E. J. McShane.

The Council adjourned at 11:30 P.M.

The Business Meeting was convened at 1:15 P.M. on January 24, 1970 in the Theater of the San Antonio Convention Center. Vice President Coxeter presided.

The Secretary reported on various matters of Council business of 
interest to the membership, as detailed in the report on the Council meeting above.

An amendment to the By-laws, already approved by the Council, was presented for action. It consists of adding italicized words to the fifth sentence of Article $X$, Section 1, so that the sentence reads as follows:

A business meeting of the Society shall take final action only on business accepted by unanimous consent, or business notified to the full membership of the Society in the call for the meeting, except that the business meetings held at either the Annual Meeting or at the Summer Meeting may take final action on business which has been recommended for consideration by the Council and has been accepted by the vote of four-fifths of the members of the Society present and voting at such a meeting.

The Business Meeting approved the amendment.

There were questions and comments on the supply and demand for mathematicians with Ph.D. degrees and on the operation of the Employment Register.

The possibility of air mailing offprints for the Mathematical Offprint Service and of air mailing the Contents of Contemporary Mathematics Journals to those willing to pay for the service was explored.

The meeting adjourned at 2:05 P.M.

Bethlehem, Pennsyluania

Tallahassee, Florida
Everett Pitcher

Secretary

Orville G. Harrold, JR. Associate Secretary

\section{THE NOVEMBER MEETING IN ANN ARBOR}

The six hundred seventy-first meeting of the American Mathematical Society was held at the University of Michigan, Ann Arbor, Michigan on Saturday, November 29, 1969. There were 238 registrants, including 201 members of the Society.

By invitation of the Committee to Select Hour Speakers for Western Sectional Meetings there were two one-hour addresses. Dr. Alan Baker of Cambridge University and the Universities of Michigan and Colorado addressed the Society on the subject $A$ survey of recent results in the theory of diophantine equations; he was introduced by the Associate Secretary. Professor Avner Friedman of Northwestern University spoke on the topic Free boundary problems for parabolic equations; Professor Tsuan Wu Ting presided at Professor Friedman's lecture. 